MATHEMATICS OF COMPUTATION

Volume 70, Number 235, Pages 1329-1333

S 0025-5718(00)01279-5

Article electronically published on October 17, 2000

\title{
BREEDING AMICABLE NUMBERS IN ABUNDANCE. II
}

\author{
STEFAN BATTIATO AND WALTER BORHO
}

\begin{abstract}
In a first article of this title, new procedures were described to compute many amicable numbers by "breeding" them in several generations. An extensive computer search was later performed (in 1988), and demonstrated the remarkable effectiveness of this breeding method: the number of known amicable pairs was easily quadrupled by this search. As we learnt recently (1999) from the internet, Pederson and te Riele have again multiplied that number roughly by ten. While they give no information on their method of search, we publish here our method and summarize the computations. Our results provide some evidence for the conjecture that the number of amicable pairs is infinite.
\end{abstract}

1

Two numbers $n$ and $m$ are amicable, iff the sum of the proper divisors of $n$ is $m$ and conversely. The first example, 220 and 284, is attributed to Pythagoras.

The method of bilinear diophantine equations (BDE-method) was used in the past by E.J. Lee [5] and many others, in special cases already by L. Euler around 1750 , to produce many amicable pairs of the form $a_{1} r_{1} r_{2}, a_{2} r_{3}$ from suitable inputs $a_{1}, a_{2}$. Here $r_{1} \neq r_{2}, r_{3}$ are three primes prime to $a_{1}, a_{2}$.

The great art of the great number hunters like Euler, Poulet, Escott, Lee was to find suitable inputs $a_{1}, a_{2}$.

The BDE-method (explained below) is modified and extended here to the "breeding method" 2. Here a breeder is a pair of positive integers $a_{1}, a_{2}$ such that

$$
\sigma\left(a_{1}\right)\left(r_{1}+1\right)=a_{1} r_{1}+a_{2} r_{2}=\sigma\left(a_{2}\right)\left(r_{2}+1\right)
$$

has a solution in positive integers $r_{1}, r_{2}$. Here $\sigma$ denotes the sum of divisors. Note that if $r_{1}, r_{2}$ are primes prime to $a_{1}, a_{2}$, then these equations mean that $a_{1} r_{1}, a_{2} r_{2}$ are amicable.

Obviously for the experts, breeders are very good inputs for the BDE-method. But another point of the breeder method is that the same BDE-method also yields new breeders as output as a by-product. So the procedure can be iterated to produce various generations of breeders, and simultaneously, amicable number pairs are produced as the usual output of the BDE-method. The overwhelming success of numerical examples (see below) demonstrates how effective this breeder method is producing breeders and simultaneously amicable pairs in abundance.

Received by the editor August 25, 1999.

2000 Mathematics Subject Classification. Primary 11A25.

(C)2000 American Mathematical Society 
In more detail following [2, 2.1], for an input pair $a_{1}, a_{2}$, e.g., a breeder of generation $n$, the following procedure is carried out: If the determinant $D:=$ $a_{1} a_{2}-\left(\sigma\left(a_{1}\right)-a_{1}\right)\left(\sigma\left(a_{2}\right)-a_{2}\right)$ is positive, the number

$$
C:=\sigma\left(a_{2}\right)\left(D\left(a_{2}-a_{1}\right)+a_{1}^{2} \sigma\left(a_{2}\right)\right)
$$

(which coincides with [2] 2.1, (21)], up to change of notation) is computed and completely factorized. Common divisors like the greatest common divisors $\left(a_{1}, \sigma\left(a_{1}\right)\right)$ and $\left(\left(\sigma\left(a_{1}\right), \sigma\left(a_{2}\right)\right)\right.$ are cancelled in the sequel. The cancellation is indicated by a bar " $\rightarrow$. If $\left(a_{1}, \sigma\left(a_{1}\right)\right)$ does not divide $a_{2}$, or if $\left(a_{2}, \sigma\left(a_{2}\right)\right)$ does not divide $a_{1}$, then the start-value $\left(a_{1}, a_{2}\right)$ is "sterile" in the sense that it gives no amicable pairs. So in this case, the procedure is stopped at this point. For each factorization $C=d_{1} d_{2}$ the numbers

$$
r_{i}:=\left(a_{1} \sigma\left(a_{2}\right)+d_{i}\right) / D-1
$$

are computed for $i=1,2$ and, if integer, are tested for primality. Whenever $r_{1}$ is prime and prime to $a_{1}$, then a breeder $\left(a_{1} r_{1}, a_{2}\right)$ of the next generation $n+1$ is found. These are the first outputs. Whenever $r_{1}, r_{2}$ and

$$
r_{3}:=\sigma\left(a_{1}\right)\left(r_{1}+1\right)\left(r_{2}+1\right) / \sigma\left(a_{2}\right)-1
$$

are simultaneously prime, different, and prime to $a_{i}$, then an amicable pair $\left(a_{1} r_{1} r_{2}, a_{2} r_{3}\right)$ of generation $n$ is found. This gives the second outputs. After finishing this procedure, the same is carried out for the input $a_{2}, a_{1}$ (in reversed order). Then the computation of generation $n+1$ is completed. Next the generation $n+2$ is computed in the same way from all breeders of generation $n+1$ as new inputs, as far as possible (i.e., as far as no overflows occur). This procedure is iterated as far as possible.

For example, take start-values $\left(a_{1}, a_{2}\right)=(4,4)$. Then, as the first generation of breeders, the four pairs $(4,4 p)$ with $p=5,7,11,19$ result. These breed 18 breeders in second generation, viz.

$\begin{array}{lllll}(4,4 \cdot 5 \cdot 11) & (4,4 \cdot 5 \cdot 13) & (4,4 \cdot 5 \cdot 101) & (4 \cdot 5,4 \cdot 13) & (4 \cdot 5,4 \cdot 17) \\ (4 \cdot 5,4 \cdot 19) & (4 \cdot 5,4 \cdot 23) & (4 \cdot 5,4 \cdot 43) & (4 \cdot 5,4 \cdot 59) & (4 \cdot 5,4 \cdot 107) \\ (4 \cdot 7,4 \cdot 11) & (4 \cdot 11,4 \cdot 11) & (4 \cdot 11,4 \cdot 13) & (4 \cdot 11,4 \cdot 17) & (4 \cdot 11,4 \cdot 29) \\ (4 \cdot 11,4 \cdot 37) & (4 \cdot 11,4 \cdot 53) & (4 \cdot 11,4 \cdot 101) & & \end{array}$

Again, these 18 breeders lead to 56 breeders in third generation. We further computed completely the fourth and fifth generation and, not quite completely (because of overflows), the sixth generation. The results follow.

\begin{tabular}{rc|c|c|c|c|c|c} 
generation: & 0 & 1 & 2 & 3 & 4 & 5 & 6 \\
\hline number of breeders: & 4 & 18 & 56 & 208 & 874 & $\geq 4686$ \\
\hline $\begin{array}{r}\text { number of amicable } \\
\text { pairs found: }\end{array}$ & 1 & 2 & 1 & 3 & 8 & $\geq 2$ & $\geq 0$
\end{tabular}

Note that the list of 17 amicable pairs found here begins with Pythagoras' pair $(220,284)$ which occurs in generation 0 . Note also that the number of breeders seems to grow exponentially, while this is not yet clear for the number of amicable pairs found. 
The breeder method can be started with any number pair $a_{1}, a_{2}$, but only a few are successful. In a first extensive search, start-values $a_{1}=a_{2}=a$ were chosen. In the range $a \leq 50000000$ only those $a$ were taken, for which the "cancelled determinant" $\bar{D}=(2 a-\sigma(a)) /(a, \sigma(a))$ had a small value $<20$. There are exactly 376 such numbers $a$ which produce at least one breeder in the second generation. For each of these 376 start-values, the above breeder algorithm was applied as far as no overflows (see below) occurred, usually up to the fourth or third generation. For each of these 376 start-values, the breeders and amicable pairs were (computed and) counted in each generation. These numbers were tabulated in the first author's Diplom-Arbeit 1] (unpublished). The table is too extensive to be reproduced here, but we do give these data in Table 1 for those 20 start-values $a$ which were most productive, i.e., which bred the largest numbers of amicable pairs. The figures in the table indicate that for these start-values the number of resulting breeders, resp. amicable pairs, seem often to grow explosively (exponentially or even more) from generation to generation. We expect that this tendency would continue, if one could compute some even higher generations.

Note that the higher generations are only partially computed here because of overflows. The general limit for numbers processed in our search was $10^{29}$ (but the resulting amicable pairs were often larger). Whenever this limit was exceeded, e.g., by $\bar{C}$ or $r_{1}, r_{2}, r_{3}$, the algorithm was stopped and the search was continued with the next items. Factorizations of $\bar{C}$ were fully carried out only up to prime factors $\leq 10^{8}$, so sometimes some factorizations may have been missed by overflow of this limit.

From Table 1 we see that the most successful start-value was $a=3^{2} \cdot 7^{2} \cdot 11 \cdot 13 \cdot 79$, which produced 5428 amicable pairs by our algorithm, that is more than $10 \%$ of the total number of amicable pairs known then (in 1988). Of these 5428 pairs, 5287 were produced by only five breeders.

Example. The champion breeder was

$$
\left(a_{1}, a_{2}\right)=\left(3^{2} \cdot 7^{2} \cdot 11 \cdot 13 \cdot 79, \quad 3^{2} \cdot 7^{2} \cdot 11 \cdot 13 \cdot 79 \cdot 1013 \cdot 6180283 \cdot 2091919367\right) .
$$

In the BDE-method for this input, one had to factorize

$$
\bar{C}=2^{19} \cdot 3^{4} \cdot 5 \cdot 7^{3} \cdot 11^{3} \cdot 13^{4} \cdot 19 \cdot 23^{4} \cdot 31^{2} \cdot 79 \cdot 89^{2} \cdot 197^{2}
$$

into two factors. Of the 4320000 factorizations, 4083964 could be fully processed and led to 2910 amicable pairs!

\section{7}

The whole search for the above-mentioned 376 start-values $a$ produced a total of almost a million breeders (most of which are too large for the amicable pair search within our limits) and a total of 26684 amicable pairs. Note that more than half of these (13996) were produced by the 20 best start-values as given in Table 1 . Together with other searches, which we do not describe here in detail, a total of 42900 amicable pairs were bred. These pairs were listed and sorted, and compared with te Riele's list of all ca. 13760 amicable pairs known in 1987 (most of them in [3]). As a result, a total of 37803 of those amicable pairs were new in 1988 (see Battiato's Diplom-Arbeit [1]). 
TABLE 1 . The 20 best inputs $a \leq 5 \cdot 10^{7}$, with $\bar{D}<20$, producing the most amicable pairs by our iterated breeding process as described in the text. For each generation (0 to 4 ) the upper entry given is the number of breeders and the lower entry is the number of amicable pairs produced. A " $\geq$ " sign indicates that this generation was only partially processed because of overflows. A "-" sign means the generation was not computed.

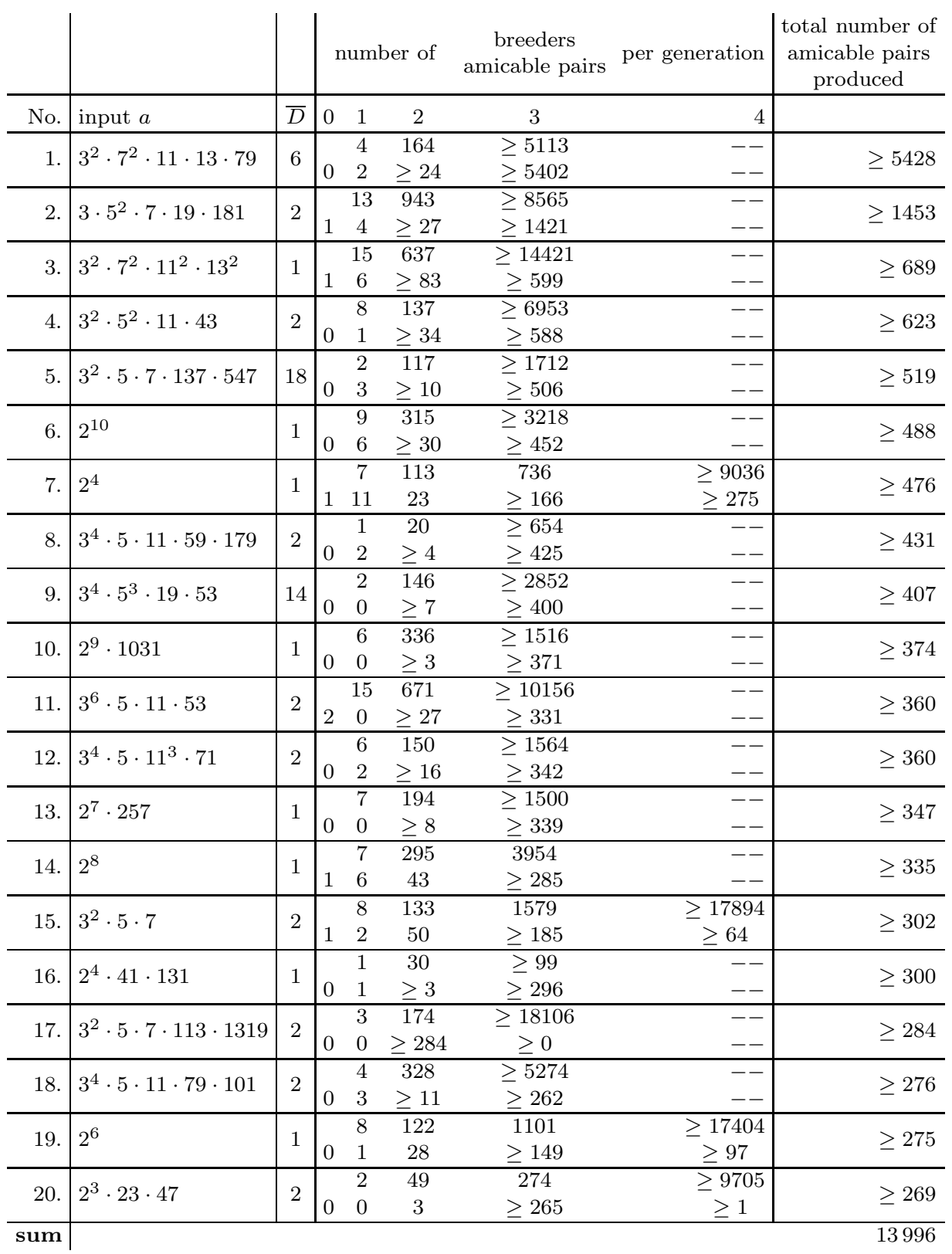


The complete list of new amicable pairs was then sent to Herman te Riele in Amsterdam, who used to keep track of all new discoveries all over the world for years. Te Riele confirmed the list. Meanwhile, the total of ca. 51560 amicable pairs thus known in 1988 was again roughly multiplied by ten by Pederson and te Riele and others, as we learnt from the internet (August 1999), address: http://www.vejlehs.dk/staff/jmp/aliquot/knwnap.htm.

Let us mention that our breeder method was also of use in finding the first odd amicable pairs not divisible by three [4], although in this case the first difficulty was to find the common factor $a$. Meanwhile, even amicable numbers prime to 30 have been found (see the internet).

\section{8}

Table 1 provides some modest evidence for the following conjecture.

Conjecture 1. For some start-values $a$ the number of breeders resp. amicable pairs produced by our algorithm in generation $n$ increases at least exponentially with $n$.

This is a very specific version of saying:

Conjecture 2. The number of amicable pairs is infinite.

\section{REFERENCES}

[1] Battiato, S.: Über die Produktion von 37803 neuen befreundeten Zahlenpaaren mit der Brütermethode, Diplom-Arbeit Wuppertal 1988 (unpublished).

[2] Borho, W. and Hoffmann, H.: Breeding amicable numbers in abundance, Math. Comp. 46 (1986), 281-293. MR 87c:11003

[3] te Riele, H. et.al: Table of amicable pairs between $10^{10}$ and $10^{52}$, Centrum voor Wiskunde en Informatica, Note NM-N8GO3, Sept. 1986.

[4] Battiato, S. and Borho, W.: Are there odd amicable numbers not divisible by three, Math. Comp. 50 (1988), 633-637. MR 89c:11015

[5] Lee, E. J.: Amicable numbers and the bilinear diophantine equation, Math. Comp. 22 (1968), 181-187. MR 37:142

Sudermannstr. 2A, 40721 Hilden, Germany

Bugh FB7, Gaussstrasse 20, 42097 Wuppertal, Germany 